\title{
Videocirurgia no Manejo da Doença de Crohn Intestinal
}

\author{
Laparoscopic Surgery in the Management of Chron's Disease
}

\author{
SERGIOEDUARDOALONSOARAÚJO ${ }^{1}$, ANDRE RONCONDIAS ${ }^{2}$, VICTOR EDMOND SEID ${ }^{3}$, FÁBIO GUILHERME \\ CAMPOS $^{4}$ E SERGIO CARLOS NAHAS $^{5}$
}

\begin{abstract}
1. Doutor em Medicina pela FMUSP. Cirurgião do Serviço de Cirurgia de Cólon e Reto do Hospital das Clínicas da FMUSP; ${ }^{2}$ Cirurgião do Serviço de Cirurgia de Cólon e Reto do Hospital das Clínicas da FMUSP; ${ }^{3}$ Mestre em Medicina pela FMUSP. Cirurgião do Serviço de Cirurgia de Cólon e Reto do Hospital das Clínicas da FMUSP;

4. Professor Livre-Docente pela Faculdade de Medicina da USP. Cirurgião do Serviço de Cirurgia de Cólon e Reto do Hospital das Clínicas da FMUSP; ${ }^{5}$ Professor Livre-Docente pela Faculdade de Medicina da USP. Diretor do Serviço de Cirurgia de Cólon e Reto do Hospital das Clínicas da FMUSP.
\end{abstract}

\begin{abstract}
ARAÚJO SEA; DIAS AR; SEID VE; CAMPOS FG; NAHAS SC. Videocirurgia no Manejo da Doença de Crohn Intestinal. Rev bras Coloproct, 2010;30(3): 265-271.
\end{abstract}

RESUMO: A doença de Crohn é uma moléstia com um amplo espectro de manifestações. Seu tratamento é complexo e frequientemente os pacientes portadores desta afecção necessitam de intervenções cirúrgicas. Com o surgimento da laparoscopia e sua popularização no tratamento das afecções intestinais, demonstrando resultados superiores ao acesso convencional e quebrando paradigmas como sua utilização no tratamento do câncer colorretal, passou-se a cogitar se esse acesso seria indicado também nas doenças inflamatórias intestinais. Ainda hoje, a utilização desta via de acesso na doença de Crohn é tema controverso. Devido à natureza inflamatória desta patologia, o grau de dificuldade cirúrgico está aumentado e muitas dúvidas persistem: há benefício para o paciente? A taxa de conversão não está exageradamente aumentada? É possível indicar esse acesso em casos complicados? Qual o grupo de pacientes que se beneficia da técnica? Nesta revisão apresentamos os dados mais recentes e as evidências científicas que sustentam a indicação da via de acesso laparoscópica no tratamento cirúrgico da doença de Crohn.

Descritores: Doença de Crohn, Doença inflamatória intestinal, Laparoscopia.

\section{INTRODUÇÃO}

A doença de Crohn (DC) têm se demonstrado particularmente desafiadora para o videocirurgião com alta demanda técnica devido à sua complicada natureza inflamatória ${ }^{1}$. No entanto, refinamentos na técnica e tecnologia levaram a consideráveis avanços e a uma definição clara do papel do procedimento laparoscópico na DC.

Os pacientes com Crohn são freqüentemente jovens (15-35 anos), conscientes de sua imagem corporal e, geralmente, resistentes à cirurgia, antecipado o desconforto e a modificação na imagem corporal, a necessidade de múltiplas cirurgias, o tempo perdido de 
trabalho e de atividades escolares, além do medo de comprometimento da função intestinal e de um possível estoma ${ }^{2}$. Neste contexto o acesso por vídeo representa uma alternativa atraente nessa população.

$\mathrm{O}$ acesso laparoscópico nos pacientes com DC é possivelmente superior à técnica aberta em casos selecionados e alguns cirurgiões experientes têm utilizado essa via de acesso inclusive em casos complicados com obstrução e fistulas. Em média, 70\% dos pacientes com DC irão necessitar de pelo menos uma operação para sua doença ${ }^{3}$, enquanto $45 \%$ irão necessitar de cirurgias repetidas ao longo de suas vidas ${ }^{4}$.

Na DC, o número de pacientes submetidos a tratamento cirúrgico avança anualmente de forma significativa nos Estados Unidos 5 . Independentemente do acesso cirúrgico, o desafio é grande, pois os pacientes podem estar comprometidos nutricionalmente e em sua imunidade. Além disso, muitas vezes possuem cirurgias abdominais prévias ou estão em condições clínicas ruins com reserva limitada. O cirurgião também precisa estar preparado para se defrontar com achados cirúrgicos inesperados, como espessamento mesenterial, friabilidade e maior tendência a sangramento, todos resultantes da inflamação crônica, estenose, abscesso, fistulas. Estes achados podem ocorrer separadamente ou em conjunto o que dificulta o trabalho do videocirurgião. A combinação de um alto índice de suspeita com a adequada investigação préoperatória por imagem ajudam a prever essas ocorrências e a planejar adequadamente a cirurgia.

\section{Indicações}

A opção de oferecer cirurgia minimamente invasiva aos pacientes com DC é válida, tem resultados conhecidos, beneficia os pacientes e sempre que possível deve ser utilizada. A videolaparoscopia é uma via de acesso cirúrgica minimamente invasiva assim como a videocirurgia com assistência manual (HALS, do inglês hand-assisted laparoscopic surgery) ou as mais recentes: videocirurgia por portal único (singleport laparoscopic surgery) ou por incisão única (single incision laparoscopic surgery).

A cirurgia a ser realizada e as indicações de tratamento cirúrgico são as mesmas e independem da via de acesso a ser empregada. Assim, as principais indicações para cirurgia laparoscópica na DC são: doença ileocólica recorrente e primária; estenoses isoladas do intestino delgado ou grosso; sepse anorretal; retardo de crescimento; obstrução por estenose ou aderência; inflamação pancólica; dor recorrente ou persistente; necessidade de restabelecimento da continuidade intestinal.

\section{Contra-indicações}

Contra-indicações relativas à laparoscopia incluem doença aguda grave, aderências múltiplas ou densas, impossibilidade de identificar a anatomia normal, perfuração não-bloqueada, peritonite difusa, grande abscesso ou flegmão e fistulas complexas múltiplas. Entretanto, as contra-indicações diminuem conforme evoluem a perícia cirúrgica e os equipamentos ${ }^{6}$.

\section{TÉCNICA OPERATÓRIA}

Uma vez indicado o tratamento cirúrgico é importante determinar a extensão da ressecção e a anastomose a ser realizada. Margens de $2 \mathrm{~cm}$ são suficientes, ressecções extensas não reduzem o risco de recidiva, além de trazer o risco de intestino curto ${ }^{7}$. Apenas segmentos macroscopicamente acometidos devem ser ressecados, não havendo vantagem em realizar biópsias de congelação para avaliar a margem ${ }^{8}$.

A respeito da anastomose no intestino delgado nossa opção pessoal é pela anastomose mecânica látero-lateral em função da menor contaminação e maior extensão da anastomose, potencialmente associada a menor risco de estenose necessitando de reoperação, ainda que este benefício não tenha sido demonstrado ${ }^{9}$. Também está comprovado qual a melhor anastomose após ressecções íleocólicas. Alguns autores mostram uma maior associação entre a anastomose término-terminal e a ocorrência de recidiva ${ }^{10,11,12}$, enquanto outros não observaram benefício na anastomose látero-lateral ${ }^{13,14}$. Um estudo randomizado comparou essas duas opções e não notou diferença estatisticamente significante quanto às recidivas endoscópica e sintomática ${ }^{15}$. Independente disto, a preferência deve ser pela sutura mecânica, uma metanálise recente avaliou os dados de 955 pacientes submetidos à ressecção ileocecal (câncer e doença de Crohn) e observou que a anastomose mecânica esteve significativamente associada a menor risco de deiscência ${ }^{16}$.

O grau de dificuldade da cirurgia na DC está aumentado. Inflamação transmural, a marca característica da doença de Crohn, resulta em fragilidade e friabilidade mesentérica, massas e adesões inflamató- 
rias, fístula e abscesso. Fístulas entéricas e massas inflamatórias associadas distorcem a anatomia normal e aumentam o risco de lesão a estruturas vitais fazendo da cirurgia um desafio mesmo para cirurgiões experientes. Além disso, nem sempre é possível retirar uma enorme massa inflamatória por uma incisão "cosmética".

As taxas de conversão variam de $2 \%$ a $77 \%$ e dependem principalmente da experiência do cirurgião ${ }^{17}$. Fatores relacionados ao paciente (obesidade, estado cardiopulmonar e laparotomias prévias) e à doença (flegmão, fístula ou abscessos, localização) também influenciam ${ }^{18}$

Com relação ao preparo mecânico intestinal, a sua realização no pré-operatório depende do tipo de operação a se realizada, podendo ser dispensado em ressecções do delgado e nas operações de ressecção ileocecal.

A antibioticoterapia de amplo espectro deve ser empregada sempre, sendo iniciada na indução anestésica e continuada durante a internação hospitalar. A profilaxia antitrombótica também é indicada rotineiramente, incluindo o uso de meias elásticas de compressão intermitente nos membros inferiores e de heparina de baixo peso molecular com a primeira dose realizada oito horas após o término da cirurgia.

A técnica anestésica está sempre a cargo do anestesiologista e favorece-se o emprego da anestesia geral sem bloqueio em nossa prática. A drenagem gástrica e vesical é sempre empregada, sendo retirada ao final da cirurgia se possível.

O pneumoperitônio é realizado pela técnica fechada com emprego de agulha de Veress descartável. Nos pacientes com cirurgia abdominal prévia com incisões abaixo da cicatriz umbilical, empregamos a mesma técnica com a punção no hipocôndrio esquerdo.

Em todas as operações colorretais por vídeo, favorecemos o posicionamento do paciente em decúbito dorsal com as pernas apoiadas em perneiras (posição de semilitotomia). Os dois membros superiores são posicionados ao longo do tronco.

O número de portais de trabalho e a abordagem cólica varia de acordo com a preferência e experiência pessoais. Nossa opção é por utilizar cinco trocartes e dissecar o cólon no sentido mediolateral, iniciando pela identificação e controle vascular, seguida da mobilização do mesocólon e reflexões peritoneais.
Aspectos técnicos da ressecção ileocecal com ou sem enterectomia por videolaparoscopia

Após a passagem dos trocartes, inicia-se o inventário da cavidade visando identificar áreas acometidas nos intestinos delgado e grosso. Ao se observar acometimento do delgado, o(s) segmento(s) de comprometido (s) é (são) assinalado(s) pela aplicação de um ponto seromuscular que permitirá sua identificação e tratamento (enteroplastia ou enterectomia) no tempo aberto da cirurgia. Como não há benefício associado à linfadenectomia, a artéria ileocólica pode ser tomada de forma rápida e confortável no meio da "tenda" que se forma como resultado da tração cecal pelo cirurgião assistente. A artéria pode ser separada da veia por dissecção e ambos vasos podem ser seccionados após ligadura com clipes metálicos (quando se encontram calibrosos) ou seladas exclusivamente com o emprego de um bisturi harmônico ou bipolar (possível na maioria dos casos).

A dissecção prossegue de medial para lateral, utilizando como parâmetro da a segunda porção do duodeno que é retroperitoneal. Não há necessidade de controle vascular de nenhum outro vaso na realização da ressecção ileocecal. O cólon é liberado do retroperitôneo até a terceira porção duodenal, sendo então solto do ligamento gastrocólico, preservando-se o epíplon. O íleo terminal é mobilizado, bem como o cólon direito junto a goteira parietocólica e flexura hepática. Quando a mobilização é obtida, o intestino é exteriorizado através de uma pequena incisão $(4 \mathrm{~cm})$ na linha média, ao nível da cicatriz umbilical. Grandes massas no íleo podem demandar incisões maiores. Através da incisão, pratica-se a secção do íleo terminal e do ascendente e a construção de anastomose mecânica látero-lateral isoperistáltica pelo disparo de um grampeador linear de 75 ou $80 \mathrm{~mm}$. A brecha do grampeador é fechada em 2 planos, e a brecha mesenterial também é obliterada. Eventuais alças de delgado previamente marcadas são exteriorizadas e tratadas. Em casos selecionados como nas pacientes jovens do sexo feminino ou nos doentes muito obesos, pode-se realizar a anastomose intracorpórea empregando técnica de endogrampeamento e endossutura. Nesses casos a peça pode ser retirada através de uma incisão cosmeticamente superior (Pfannestiel). Entretanto, esta opção é mais difícil tecnicamente, onerosa e demorada.

Vale lembrar que devido à sua natureza inflamatória a DC traz um maior risco de sangramento e o 
uso de fontes diferenciadas de energia para a realização da dissecção e hemostasia, como o eletrocautério bipolar ou o bisturi harmônico facilitam e agilizam a cirurgia, além de diminuir a perda sanguínea.

Aspectos técnicos da colectomia total, da retocolectomia total e da operação de bolsa ileal por videolaparoscopia

Nas operações de colectomia total e retocolectomia total assistidas por laparoscopia, nossa preferência pessoal também é pela dissecção no sentido medial para lateral, iniciando a abordagem com a desvascularização do cólon direito e do transverso, seguida da mobilização de ambos. Posteriormente, mudamos o posicionamento da equipe e dos equipamentos, prosseguindo com a ligadura vascular da veia mesentérica inferior e da artéria mesentérica inferior seguidas da mobilização do ângulo esplênico e do cólon esquerdo. Em todos os momentos de ligadura vascular, esta é feita longe da origem vascular, pois se trata de ressecção cólica por doença benigna. A dissecção do reto por que precede a construção de ileostomia terminal definitiva (ou muito raramente, de reservatório ileal) obedece ao princípio de preservação autonômica, mas não ao de excisão total do mesorreto. Dessa forma, a dissecção da porção "horizontal" do reto é feita mais próxima da muscular própria do reto e longe do arcabouço pélvico. Sempre que possível, a proctectomia é realizada no plano inter-esfincteriano, poupando-se tecido perineal, diferentemente da amputação do reto com fins oncológicos.

\section{RESULTADOS DA VIDEOCIRURGIA NA DOENÇA DE CROHN}

Técnicas laparoscópicas têm sido aplicadas a pacientes com doença de Crohn desde o início dos anos 90. Contudo, assim como outras indicações para cirurgia colorretal por vídeo, esse acesso não têm sido universalmente aceito. Aderências, flegmão e trajetos fistulosos podem representar desafios técnicos significantes para o cirurgião, aumentando o tempo operatório e a taxa de conversão. Apesar disso, veremos a seguir que um número substancial de pacientes se beneficia da cirurgia laparoscópica.

As necessidades cirúrgicas na doença de Crohn variam de acordo com o espectro da doença e suas complicações. Neste sentido a laparoscopia não difere da cirurgia aberta e vem apenas adicionar a oportunidade de buscarmos um melhor tratamento para o paciente.

Vale citar que apesar do grande número de publicações disponíveis, demonstrando, por exemplo, que as ressecções íleocólicas vídeo-assistidas são factíveis e seguras a factibilidade no tratamento da doença de Crohn, a maioria destas experiências não têm grupo controle ou consiste de ensaios não randomizados $19,20,21,22,23,24,25,26,27,28,29$.

Quanto à dificuldade técnica, procedimentos simples como a laparoscopia diagnóstica e a derivação fecal são bons procedimentos iniciais e requerem equipamento mínimo e podendo ser realizados por cirurgiões com experiência limitada com a cirurgia laparoscópica.

Comparando a derivação fecal laparoscópica com a aberta a primeira permite um tempo operatório menor, com retorno mais precoce da função intestinal e menor duração da internação hospitalar ${ }^{26,30}$.

A ressecção ileocecal ou ileocólica é o procedimento laparoscópico mais comumente realizado. A primeira grande série de pacientes com doença inflamatória intestinal operados por laparoscopia data de 1996. No total 49 pacientes com DC e 23 com retocolite ulcerativa foram tratados. A ressecção íleocólica laparoscópica foi realizada em 30 pacientes com taxa de morbidade de $10 \%$. O tempo operatório médio foi de 150 min e o tempo médio de internação hospitalar foi de 5,2 dias ${ }^{31}$.

Em 1997, Wu e cols ${ }^{22}$ demonstraram que abscessos, flegmão e recidivas não são contra-indicações para a cirurgia laparoscópica. Chen e cols ${ }^{25}$ demonstraram que o tempo médio até o retorno às atividades parciais é menor nos pacientes com Crohn tratados pela via laparoscópica, quando comparados à via aberta $(2,1 \pm 1,2$ versus $4,4 \pm 2,8$ semanas; $p<0,0001)$. $O$ retorno para atividade completa foi também menor no grupo laparoscópico (4,2 $\pm 2,3$ versus 10,5 $\pm 6,4$ semanas; $\mathrm{p}<0,0001)$, assim como o retorno ao trabalho $(3,7$ $\pm 2,1$ versus 7,5 $\pm 7,5$ semanas; $p=0,01$ ).

Ao comparar pacientes submetidos à ressecção íleocólica vídeo-assistida, com outros submetidos à via aberta, notamos que a via laparoscópica tem maior tempo operatório, menor de internação e superioridade cosmética ${ }^{32,33,34}$. Além disso, alguns autores ainda relatam uma menor morbidade nos pacientes submetidos à via laparoscópica ${ }^{35} \mathrm{Um}$ estudo prospectivo, randomizado e multicêntrico observou ain- 
da que há redução dos custos do tratamento quando a laparoscopia é realizada ${ }^{36}$.

Estudos mais recentes que os acima citados têm confirmado os benefícios globais da laparoscopia no tratamento da DC, com vantagens na resolução do íleo pós-operatório e no tempo de internação $37,38,39,40,41,42$.

Até recentemente os resultados em longo prazo da ressecção íleocólica laparoscópica em pacientes com DC eram desconhecidos. Em 2006, Lowney e cols ${ }^{30}$ observaram que não há diferença quanto à recidiva em pacientes submetidos à ressecção íleocólica por vídeo ou convencional.

Uma metanálise de 14 estudos, publicada em 2007, incluiu 881 pacientes com DC operados pela via convencional ou laparoscópica. O estudo concluiu que i. as operações por vídeo são mais demoradas em cerca de 30 minutos; ii. a duração do íleo pós-operatório foi 0,75 dias menor no grupo operado por vídeo; iii. a internação hospitalar foi em média 1,82 dias menor no grupo operado por vídeo; iv. o risco de uma complicação associada à via de acesso é $50 \%$ na vídeocirurgia. Os resultados dessa metanálise subsidiam as conclusões de que os pacientes operados por videolaparoscopia são selecionados e que são operados com menor morbidade e menor duração da internação, às custas de um tempo operatório maior $^{43}$.

A despeito de a evidência científica disponível demonstrar que a vídeocirurgia é empregada em casos selecionados, já existem dados acerca do seu uso na doença complicada. As evidências provêm de séries com reduzida casuística, porém algumas conclusões podem ser obtidas como se depreende da análise da Tabela 1. A duração da cirurgia, da internação e a morbidade nesses pacientes estão significativamente aumentadas, mas a cirurgia nesses casos é mais difícil e demorada e a conversão ou pela opção de laparotomia desde o início não necessariamente mudaria esses resultados. A taxa de conversão também está aumentada. Em suma, o manejo laparoscópico de pacientes com doença de Crohn complicada também é factível e seguro, o que leva a crer que grupos experientes em cirurgia minimamente invasiva podem oferecer essa opção aos seus pacientes.

Tabela 1 - Resultados da via de acesso por vídeo no manejo de pacientes com doença de Crohn complicada por fistula, abscesso ou recidiva.

\begin{tabular}{|c|c|c|c|c|c|c|c|}
\hline Autor,ano & $\mathbf{N}$ & Complicação & $\begin{array}{c}\text { Cirurgia } \\
\text { (min) }\end{array}$ & $\begin{array}{c}\text { Conversão } \\
(\%)\end{array}$ & $\begin{array}{c}\text { Mortalidade } \\
(\%)\end{array}$ & $\begin{array}{l}\text { Morbidade } \\
(\%)\end{array}$ & $\begin{array}{l}\text { Internação } \\
\text { (dias) }\end{array}$ \\
\hline Wu $1997^{22}$ & 24 & $\begin{array}{l}\text { Abscesso } \\
\text { e recidiva }\end{array}$ & 150 & 20 & 0 & 10 & 5 \\
\hline Watanabe $2002{ }^{44}$ & 20 & Fístula & $\begin{array}{l}180 \\
(114-300)\end{array}$ & 16 & 0 & 16 & $8 \quad(6-21)$ \\
\hline Hasegawa2003 23 & 16 & Recidiva & $\begin{array}{l}210 \\
(136-470)\end{array}$ & 13 & 0 & 19 & $8(6-14)$ \\
\hline Uchikoshi $20044^{45}$ & 23 & Recidiva & $\begin{array}{l}231 \\
(100-410)\end{array}$ & 70 & 0 & 13 & $16-22$ \\
\hline Moorthy2004 46 & 26 & Recidiva & 118 & 42 & 0 & 15 & 8 \\
\hline Lawes $2006^{47}$ & 14 & Recidiva & $\begin{array}{l}100 \\
(60-150)\end{array}$ & 0 & 0 & 7 & $5 \quad(3-9)$ \\
\hline
\end{tabular}

ABSTRACT: Crohn's disease has a broad spectrum of symptoms. Its management is complex frequently requiring surgical intervention. Laparoscopic surgery revolutioned the treatment of intestinal diseases, demonstrating superiority over conventional access in several pathologies, including colorectal cancer. Due to its inflammatory nature, Crohn's disease remains a barrier to laparoscopic access. Controversy and discussion still persist: Is it safe? What are the group of patients that will benefit? Aren't conversion rates extremely high? Can it be performed for complicated forms of the disease? This revision presents the most recent data avaliable concerning Crohn and laparoscopy.

Key words: Crohn's Disease, Inflammatory Bowel Disease, laparoscopy. 


\section{REFERÊNCIAS}

1- Wexner SD, Cera SM. Laparoscopic surgery for ulcerative colitis. Surg Clin N Am 2005; 85:35-47.

2- Drossman DA, Patrick DL, Mitchell CM, Zagami EA, Appelbaum MI. Health related quality of life in inflammatory bowel disease. Functional status and patient worries and concerns. Dig Dis Sci 1989; 34: 1379-86.

3- Farmer RG, Whelan G, Fazio VW. Long term follow up of patients with Crohn's disease. Relationship between the clinical pattern and prognosis. Gastroenterology 1979; 77: 907-13.

4- Kornbluth A, Sachar DB, Salomon P. Crohn's disease. In: Sleisenger M, Fordtran J, eds. Gastrointestinal and Liver Disease: Pathophysiology, Diagnosis, Management. Philadelphia: WB Saunders, 1993: 1270-304.

5- Lesperance K, Martin MJ, Lehmann R, Brounts L, Steele SR. National trends and outcomes for the surgical therapy of ileocolonic Crohn's disease: a population-based analysis of laparoscopic vs. open approaches. J Gastrointest Surg. 2009; 13(7):1251-9.

6- Goyer P, Alves A, Bretagnol F, Bouhnik Y, Valleur P, Panis Y. Impact of complex Crohn's disease on the outcome of laparoscopic ileocecal resection: a comparative clinical study in 124 patients. Dis Colon Rectum 2009; 52: 205-10.

7- Fazio VW, Marchetti F, Church M, Goldblum JR, Lavery C, Hull TL, et al. Effect of resection margins on the recurrence of Crohn's disease in the small bowel. A randomised controlled trial. Ann Surg 1996; 224: 563-71.

8- Hamilton SR, Reese J, Pennington L, Boitnott JK, Bayless TM, Cameron JL. The role of resection margin frozen section in the surgical management of Crohn's disease. Surg Gynecol Obstet 1985; 160:57-62.

9- Resegotti A, Astegiano M, Farina EC, Ciccone G, Avagnina G, Giustetto A, et al. Side-to-side stapled anastomosis strongly reduces anastomotic leak rates in Crohn's disease surgery. Dis Colon Rectum 2005; 48: 464-8.

10- Yamamoto T, Bain IM, Mylonakis E, Allan RN, Keighley MR. Stapled functional end-to-end anastomosis versus sutured end-to-end anastomosis after ileocolonic resection in Crohn disease. Scand J Gastroenterol 1999; 34: 708-13.

11- Muñoz-Juárez M, Yamamoto T, Wolff BG, Keighley MR. Wide-lumen stapled anastomosis vs. conventional end-to-end anastomosis in the treatment of Crohn's disease. Dis Colon Rectum 2001; 44: 20-5.

12- Tersigni R, Alessandroni L, Barreca M, Piovanello P, Prantera C. Does stapled functional end-to-end anastomosis affect recurrence of Crohn's disease after ileocolonic resection? Hepatogastroenterology 2003; 50: 1422-5.

13- Kusunoki M, Ikeuchi H, Yanagi H, Shoji Y, Yamamura T. A comparison of stapled and hand-sewn anastomoses in Crohn's disease. Dig Surg 1998; 15: 679-82.

14- Moskovitz D, McLeod RS, Greenberg GR, Cohen Z. Operative and environmental risk factors for recurrence of Crohn's disease. Int J Colorectal Dis 1999; 14(4-5):224-6.
15- McLeod RS, Wolff BG, Ross S, Parkes R, McKenzie M; Investigators of the CAST Trial. Recurrence of Crohn's disease after ileocolic resection is not affected by anastomotic type: results of a multicenter, randomized, controlled trial. Dis Colon Rectum 2009; 52:919-27.

16- Choy PY, Bissett IP, Docherty JG, Parry BR, Merrie AE. Stapled versus handsewn methods for ileocolic anastomoses. Cochrane Database Syst Rev 2007; 18; (3):CD004320.

17- Marusch F, Gastinger I, Schneider C, Scheidbach H, Konradt J, Bruch HP, et al. Importance of conversion for results obtained with laparoscopic colorectal surgery. Dis Colon Rectum 2001;44:207-16.

18- Marcello PW, Wong SK. Measuring outcomes of laparoscopic colectomy: is there an advantage? Semin Colon Rectal Surg 1999; 10:110-9.

19- Milsom JW, Lavery IC, Bohm B, Fazio VW. Laparoscopically assisted ileocolectomy in Crohn's disease. Surg Laparosc Endosc 1993;3: 77-80.

20- Liu CD, Rolandelli R, Ashley SW, Evans B, Shin M, McFadden DW. Laparoscopic surgery for inflammatory bowel disease. Am Surg 1995;61:1054-6.

21- Ludwig KA, Milsom JW, Church JM, Fazio VW. Preliminary experience with laparoscopic intestinal surgery for Crohn's disease. Am J Surg 1996;171:52-5.

22- Wu JS, Birnbaum EH, Kodner IJ, Fry RD, Read TE, Fleshman JW. Laparoscopic-assisted ileocolic resections in patients with Crohn's disease: are abscesses, phlegmons, or recurrent disease contraindications? Surgery 1997; 122:682-9.

23- Hasegawa H, Watanabe M, Nishibori H, Okabayashi K, Hibi T, Kitajima M. Laparoscopic surgery for recurrent Crohn's disease. Br J Surg 2003; 90:970-3.

24- Chen HH, Wexner SD, Weiss EG. Laparoscopic colectomy for benign colorectal disease is associated with a significant reduction in disability as compared to laparotomy. Surg Endosc 1998;12: 1397-400.

25- Ogunbiyi OA, Fleshman JW. Place of laparoscopic surgery in Crohn's disease. Baillieres Clin Gastroenterol 1998;12:15765.

26- Hollyoak MA, Lumley J, Stitz RW. Laparoscopic stoma formation for fecal diversion. Br J Surg 1998;85:226-8.

27- Hamel CT, Hildebrandt U, Weiss EG, Feifelz G, Wexner SD. Laparoscopic surgery for inflammatory bowel disease ileocolic resection versus subtotal colectomy. Surg Endosc 2001;15:642-5.

28- Bauer JJ, Harris MT, Grumbach NM, Gorfine SR. Laparoscopic assisted intestinal resection for Crohn's disease. Which patients are good candidates? J Clin Gastroenterol 1996;23:44-6.

29- Lowney JK, Dietz DW, Birnbaum EH, Kodner IJ, Mutch MG, Fleshman JW. Is there any difference in recurrence rates in laparoscopic ileocolic resection for Crohn's disease compared with conventional surgery? A long-term, follow-up study. Dis Colon Rectum. 2006 Jan;49(1):58-63. 
30- Iroatulam AJ, Potenti FM, Oliveira L, Pikarsky AJ, Wexner SD. Laparoscopic versus open stoma creation for fecal diversion. Tech Coloproctol 2000;4:83-7.

31- Reissman P, Salky BA, Pfeifer J, Edye M, Jagelman DG, Wexner SD. Laparoscopic surgery in the management of inflammatory bowel disease. Am J Surg 1996; 171:47-51.

32- Bemelman WA. Long-term surgical recurrence, morbidity, quality of life, and body image of laparoscopic-assisted vs. open ileocolic resection for Crohn's disease: a comparative study. Dis Colon Rectum 2008; 51: 858-67.

33- Alabaz O, Iroatulam AJ, Nessim A, Weiss EG, Nogueras JJ, Wexner SD. Comparison of laparoscopically assisted and conventional ileocolic resection for Crohn's disease. Eur J Surg 2000;166:213-7.

34- Dunker MS, Stiggelbout AM, van Hogezand RA, Ringers J, Griffioen G, Bemelman WA. Cosmesis and body image after laparoscopic-assisted and open ileocolic resection for Crohn's disease. Surg Endosc 1998;12:1334-40.

35- Milsom JW, Hammerhofer KA, Böhm B, Marcello P, Elson P, Fazio VW. Prospective, randomized trial comparing laparoscopic vs conventional surgery for refractory ileocolic Crohn's disease. Dis Colon Rectum 2001;44: 1-9.

36- Maartense S, Dunker MS, Slors JF, Cuesta MA, Pierik EG, Gouma DJ, et al. Laparoscopic-assisted versus open ileocolic resection for Crohn's disease: a randomized trial. Ann Surg. 2006 Feb;243(2):143-9; discussion 150-3.

37- Luan X, Gross E. Laparoscopic assisted surgery for Crohn's disease: an initial experience and results. J Tongji Med Univ 2000;20:332-5.

38- Bergamaschi R, Pessaux P, Arnaud JP. Comparison of conventional and laparoscopic ileocolic resection for Crohn's disease. Dis Colon Rectum 2003;46:1129-33.

39- von Allmen D, Markowitz JE, York A, Mamula P, Shepanski M, Baldassano R. Laparoscopic assisted bowel resection offers advantages over open surgery for treated segmental Crohn's disease in children. J Pediatr Surg 2003;38:963-5.
40- Shore G, Gonzalez QH, Bondora A, Vickers SM. Laparoscopic vs. conventional ileocolectomy for primary Crohn's disease. Arch Surg 2003;138:76-9.

41- Benoist S, Panis Y, Beaufour A, Bouhnik Y, Matuchansky C, Valleur P. Laparoscopic ileocecal resection in Crohn's disease: a case matched comparison with open resection. Surg Endosc 2003; 17:814-8

42- Huilgol RL, Wright CM, Solomon MJ. Laparoscopic versus open ileocolic resection for Crohn's disease. J Laparoendosc Adv Tech A 2004; 14:61-5.

43- Eshuis EJ, Polle SW, Slors JF, Hommes DW, Sprangers MA, Gouma DJ, et al. Laparoscopic surgery for Crohn's disease: a meta-analysis. Dis Colon Rectum 2007; 50: 576-85.

44- Watanabe M, Hasegawa H, Yamamoto S, Hibi T, Kitajima M. Successful application of laparoscopic surgery to the treatment of Crohn's disease with fistulas. Dis Colon Rectum. 2002 Aug;45(8):1057-61.

45- Uchikoshi F, Ito T, Nezu R, Tanemura M, Kai Y, Mizushima $\mathrm{T}$, et al. Advantages of laparoscope-assisted surgery for recurrent Crohn disease. Surg Endosc 2004; 18: 1675-9.

46- Moorthy K, Shaul T, Foley RJ. Factors that predict conversion in patients undergoing laparoscopic surgery for Crohn's disease. Am J Surg 2004; 187: 47-51.

47- Lawes DA, Motson RW. Avoidance of laparotomy for recurrent disease is a long-term benefit of laparoscopic resection for Crohn's disease. Br J Surg 2006; 93: 607-8.

\section{Endereço para correspondência:}

SERGIO EDUARDO ALONSO ARAUJO

Rua Cristiano Viana, 450.Apto 62.

Jardim América, São Paulo, SP, Brasil.

CEP: 05411-000

Fone: $+55113082-7748$

Fax: + $55113082-7748$

E-mail: sergioed@terra.com.br 